\title{
openheart Self-reported and objectively measured physical activity in people with and without chronic heart failure: UK Biobank analysis
}

\author{
Johanna O'Donnell (D) , ${ }^{1,2}$ Karl Smith-Byrne, ${ }^{3}$ Carmelo Velardo, ${ }^{2}$ \\ Nathalie Conrad (D) , ${ }^{1}$ Gholamreza Salimi-Khorshidi, ${ }^{1}$ Aiden Doherty, ${ }^{3}$ \\ Terence Dwyer, ${ }^{1}$ Lionel Tarassenko, ${ }^{2}$ Kazem Rahimi (D) ${ }^{1}$
}

\begin{abstract}
- Additional material is published online only. To view, please visit the journal online (http://dx.doi.org/10.1136/ openhrt-2019-001099).
\end{abstract}

To cite: O'Donnell J, Smith-Byrne K, Velardo C, et al. Self-reported and objectively measured physical activity in people with and without chronic heart failure: UK Biobank analysis. Open Heart 2020;7:e001099. doi:10.1136/ openhrt-2019-001099

Received 6 July 2019 Revised 18 October 2019 Accepted 27 November 2019

\section{Check for updates}

(c) Author(s) (or their employer(s)) 2020. Re-use permitted under CC BY. Published by BMJ.

${ }^{1}$ George Institute for Global Health, University of Oxford, Oxford, Oxfordshire, UK ${ }^{2}$ Institute of Biomedical Engineering, University of Oxford, Oxford, UK ${ }^{3}$ Nuffield Department of Population Health, University of Oxford, Oxford, UK

Correspondence to Dr Johanna 0'Donnell; johanna. em.odonnell@gmail.com

\section{ABSTRACT}

Objective The impact of heart failure (HF) on perceived and objectively measured levels of physical activity

(PA) can inform risk stratification and treatment recommendation. We aimed to compare self-reported and objectively measured PA levels in a large sample of participants with and without HF.

Methods A validated PA questionnaire was used to estimate self-reported weekly PA among 1600 participants with HF and 387580 participants without HF. Accelerometer data were studied in 596 participants with HF and 96105 participants without $\mathrm{HF}$ for a period of 7 days. Using multivariable linear regression models, we compared the PA levels between participants with $\mathrm{HF}$ and without HF, focusing on both the average daily PA levels and the intensity of PAs throughout the day.

Results PA levels were significantly lower in participants with HF using both self-report (excess metabolic equivalent of task hours per week of $26.5(95 \% \mathrm{Cl} 24.7$ to 28.4 ) vs 34.7 (95\% Cl 34.5 to 34.9 ), respectively $(\mathrm{p}<0.001))$ and accelerometer measures (mean accelerations of 23.7 milligravity ( $95 \% \mathrm{Cl} 23.1$ to 24.4 ) vs 28.1 milligravity $(95 \% \mathrm{Cl} 28.0$ to 28.1$)$, respectively $(p<0.001))$. Findings were consistent across different PA intensities. Hour-by-hour comparisons showed that accelerometer-derived PA levels of patients with HF were reduced throughout the day.

Conclusion Perceived and objectively recorded PA levels of patients with chronic HF are significantly lower than those of individuals without HF. This difference is continuous throughout the different hours of the day, with individuals with HF being on average $16 \%$ less active than individuals without HF. In patients with HF, increases in everyday activity may be a potential alternative to structured exercise programmes.

\section{INTRODUCTION}

In patients with heart failure (HF), increases in levels of physical activity (PA) improve quality of life ${ }^{1}$ and may reduce the risk of hospitalisation and death. ${ }^{2}{ }^{3}$ Therefore, many national and international guidelines strongly recommend regular PA and
Key messages

What is already known about this subject?

- Reduced levels of physical activity (PA) in heart failure (HF) patients have been associated with more severe levels of breathlessness and an increased risk of long-term mortality, however rlittle is known about PA levels of HF patients throughout the day and in comparison with non-HF individuals.

What does this study add?

- In this study, we compare self-reported and accelerometer-derived PA levels in a large group of individuals with and without $\mathrm{HF}$ and show that both perceived and objectively recorded PA levels of patients with chronic HF are significantly lower than those of individuals without HF. In our study, accelerometer-derived measures revealed that PA levels of patients with HF are significantly reduced throughout the day.

How might this impact on clinical practice?

- This research allows clinical staff to gain a better understanding of the impact of $\mathrm{HF}$ on patients' dayto-day life. We show that both patients self-reported (perceived) and accelerometer-derived (objective) $P A$ is significantly lower than in participants without HF when adjusting for age and sex. Furthermore, through the added detail provided by accelerometer recordings, we show that PA levels of patients with HF are significantly attenuated throughout the entire day with the exception of night hours, suggesting that HF affects the ability of patients to conduct even basic everyday PA. The latter may also suggest that increases in the activeness during everyday activities may be a potential alternative to a structured physical exercise regimen. Such interventions may be of particular benefit to patients who express concern about potential adverse effects of physical exercise.

structured exercise training for patients with mild to moderate chronic $\mathrm{HF}^{4-6}$ However, these recommendations are rarely achieved by patients. $^{7}$ 
Factors influencing the adherence to recommended PA guidelines in HF include fluctuating health and patients' external and internal motivations. ${ }^{8}$ In addition, patients' concern about the risks associated with overdoing physical exercise has been identified as a barrier to adherence $^{8}$; this might be fuelled by poor patient and doctor awareness of individuals' ability to conduct PA.

While exercise intolerance is a well-studied symptom of HF, levels of everyday PA are less well understood. ${ }^{5}$ Existing research suggests that reduced PA levels in patients with HF are associated with more severe levels of breathlessness ${ }^{67}$ and an increased risk of long-term mortality. ${ }^{9-11}$ However, reliable analysis of self-reported and objectively measured PA among patients with HF and their comparison with individuals without HF is limited. ${ }^{51213}$

This study set out to compare PA behaviour of people with and without HF using both self-reported (perceived) and accelerometer-derived (objective) measures and to investigate objectively recorded PA levels of patients with HF throughout the day.

\section{METHODS}

\section{Study population}

This research has been conducted using the UK Biobank Resource $^{14}$ (study ID 16032). Between 2006 and 2010, the UK Biobank recruited approximately 500000 subjects aged 40-69 years from across the UK and collected a range of self-reported data, physiological measurements and biological samples at baseline.

Information about health-related outcomes (before and after the baseline assessment) were obtained through linkage of the UK Biobank with data from the Office for National Statistics for death and emigration information and the Department of Health's Hospital Episode Statistics for hospital-admission diagnoses (up until March 2015 for English residents that were either NHS-funded or NHS-treated).

\section{Self-reported PA}

Baseline assessment included information on selfreported PA measured through an adapted version of the International PA questionnaire (IPAQ) ${ }^{15}$ completed on a tablet computer. Patients were asked to state how many days they were engaged in more than 10 minutes of each, walking, moderate PA and vigorous PA in a typical week. Participants were then questioned for how many minutes they were engaged in each of the activities on a typical day.

Self-reported PA data were processed using the method described by Bradbury $e t a l^{16}$ based on the IPAQ guidelines. ${ }^{17}$ Walking and moderate and vigorous activities were scored at 2.3, 3.0 and 7.0 excess metabolic equivalents of tasks (METs), respectively. ${ }^{17}$ In order to extract participants' average excess METs per week, the time spent in each of the activities on a typical day was multiplied by the typical number of days doing the exercise and the respective MET scores. Self-reported average daily physically active minutes of $<10 \mathrm{~min}$ were recorded as 0 . After aggregating over the whole week, self-reported values of $\geq 1260$ min per week (equivalent to an average of 3 hours a day) were truncated at $1260 \mathrm{~min}$ according to the IPAQ guidelines. ${ }^{16}$ Self-reported PA levels were reported in excess MET-hours per week. Participants who answered 'do not know' or 'prefer not to answer' to any of the self-reported PA questions were removed from the analysis.

\section{Accelerometer-derived PA}

Accelerometer data were collected for a subset of approximately 100000 participants between May 2013 and 2015. ${ }^{18}$ UK Biobank participants were contacted via email and provided with an Axivity AX3 accelerometer (Open Lab, Newcastle, UK) if they agreed to take part in the activity data collection. Participants were told to wear the three-axial accelerometer continuously on their dominant wrist for a period of 7 days. The accelerometer recorded data at a frequency of $100 \mathrm{~Hz}$ and an acceleration range of $\pm 8 \mathrm{~g}$.

The raw accelerometer data were calibrated ${ }^{19}$ and wear-time periods were identified using the UK Biobank preprocessing methods described by Doherty et al. ${ }^{18}$ Signals with poor wear-times or failure to calibrate were removed from the analysis. Accelerometer-based summary measures included the total mean acceleration over the 7-day measurement period, mean hourly acceleration and time spent within a range of different mean acceleration values as a marker of PA intensities. ${ }^{18}$ The proportion of time spent in sedentary, light, moderate and vigorous PAs was thereby defined as the proportion of time spent in accelerations of $\leq 25,26-100,101-425$ and $>425$ milligravity, respectively. ${ }^{1820}$

\section{HF definition}

We defined prevalent HF as any primary or secondary discharge diagnosis of $\mathrm{HF}$ as indicated by the following codes: ICD10 code 'I500', 'I501' or 'I509' and ICD9 code '4280' or '4281' prior to the assessment of PA. Given that accelerometer data were collected after questionnairebased PA assessment and were restricted to a subsample of the total cohort, we had two partially overlapping HF populations (figure 1).

\section{Covariate definitions}

Age was defined as age at the time of each PA measurement. All other covariates were based on data collected at baseline. Smoking and alcohol status were divided into never, previous or current. Social deprivation was determined using the Townsend Deprivation Index, which is an area-of-residence-based social deprivation index. ${ }^{21}$

\section{Statistical analysis}

We used multivariable linear regression to compare the mean PA between participants with HF and without HF. Analyses were carried out separately for self-reported and accelerometer-derived PA levels. Primary analyses were adjusted for age and sex. Secondary analyses were 

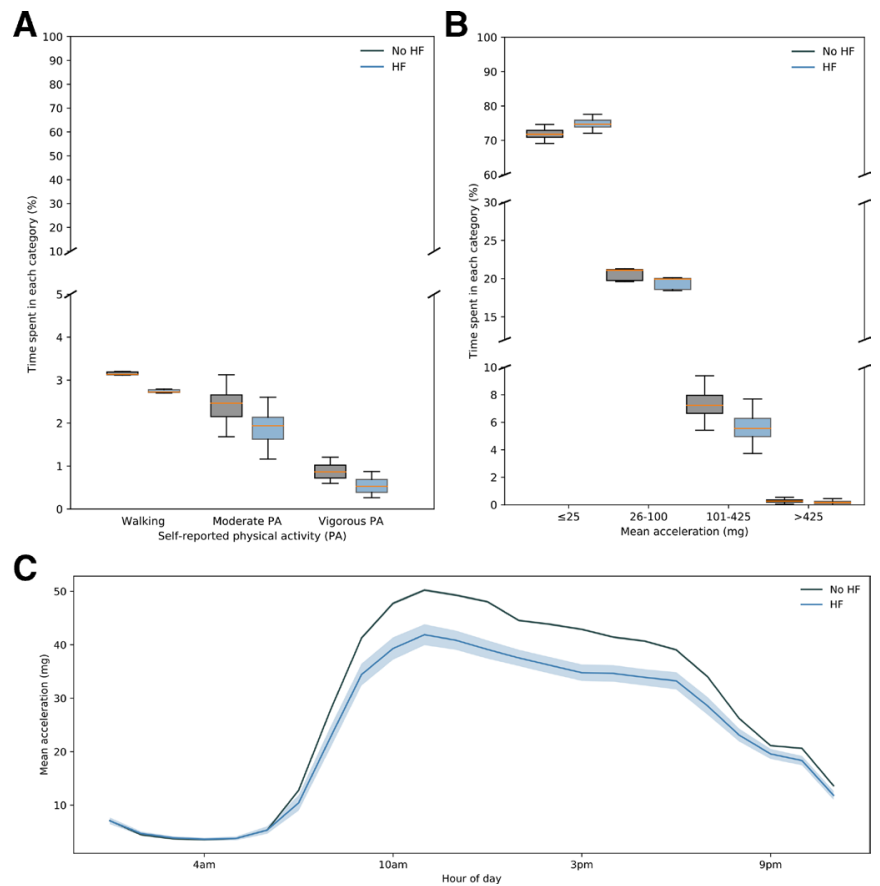

Figure 1 Summary of self-reported and accelerometerderived PA behaviours in participants with or without HF. (A) Box plots of percentage time spent within different categories of mean age-adjusted/sex-adjusted self-reported PA among participants with (blue) or without (grey) diagnosed HF. All differences are significant $(p<0.01)$. (B) Box plots of percentage time spent within different mean age-adjusted/ sex-adjusted acceleration ranges for participants with (blue) and without (grey) diagnosed HF. All differences are significant $(p<0.01)$. (C) Mean (solid line) and Cls (shaded area) of mean acceleration during different hours of the dayfor participants with (blue) and without (grey) diagnosed HF. HF, heart failure; PA, physical activity.

additionally adjusted for ethnicity, baseline Body Mass Index (BMI), alcohol status, smoking status and socioeconomic status. We performed two sensitivity analyses to test the impact of timing of HF diagnosis and PA assessment on the observed outcomes. In the first sensitivity analysis, we restricted comparisons to participants who had HF at baseline (ignoring any participants with incident HF report between baseline assessment and accelerometer data collection) to ensure that any observed differences between self-reported and objectively assessed PA levels were not due to a change in population classification over time. In a second sensitivity analysis, we investigated the impact of time since diagnosis of $\mathrm{HF}$ on any observed differences in PA levels between HF and non-HF participants. Given that both time since incident diagnosis of $\mathrm{HF}$ and time since last admission to hospital with HF could impact PA levels, we selected three time intervals as alternative explanatory variables: time since first report of HF, time since last report of HF hospitalisation where HF was the primary discharge diagnosis and time since last report of HF hospitalisation where HF was the primary or secondary discharge diagnosis. All analyses were done in $\mathrm{R}^{22}$

\section{RESULTS}

Out of a total of 502602 UK Biobank participants, 389180 provided valid self-reported PA information at baseline. This included 1600 participants with previously diagnosed HF. Accelerometer-based PA data of sufficient quality (to be included in the analysis) were collected for 96701 UK Biobank participants between 2013 and 2015, of whom 596 had an HF diagnosis prior to the recording. A subset of these participants $(\mathrm{n}=244)$ had HF at baseline; others were diagnosed with HF between the baseline assessment and the recording of accelerometer data. Out of the total of 80121 with self-reported PA data at baseline and a valid accelerometer recording (overlapping group), $190 \mathrm{had}$ a diagnosis of HF at baseline (see figure 1).

Characteristics of participants in the three subpopulations (self-reported PA, accelerometer-based PA and overlapping PA assessment) by HF status are presented in table 1 . Across all populations, patients with HF were on average older than non-HF participants and were more likely to be male. Participants with HF were more likely to be overweight or obese than participants without an HF diagnosis. HF participants were more likely to have given up alcohol and to class themselves as previous or current smokers in all analyses.

The mean age/sex-adjusted self-reported PA level per week was 26.5 (95\% CI 24.7 to 28.4) MET-hours per week for participants with diagnosed HF vs 34.7 (95\% CI 34.5 to 34.9) MET-hours per week for participants without any prior diagnosis of HF $(\mathrm{p}<0.01)$ (see figure 2$)$. The results did not change substantially when the model was further adjusted for BMI, alcohol consumption, smoking status and social deprivation score (table 2). Both times since first and last HF-related hospitalisation did not significantly affect PA levels reported by patients with HF either (table 3). According to the self-reported data, patients with $\mathrm{HF}$ spent less time in all types of reported $\mathrm{PA}$, including walking and moderate and vigorous PAs (figure 3A).

During the assessment, participants with diagnosed HF wore their accelerometers for a median of 6.9 (25th and 75th percentiles 6.8 and 7.0, respectively). Participants without HF wore the devices for an average of 6.9 (6.7 and 7.0) days. The mean acceleration values were significantly higher in participants without HF than those with HF (28.1 (95\% CI 28.0 to 28.1) milligravity vs 23.7 (95\% CI 23.1 to 24.4 ) milligravity, respectively; $\mathrm{p}<0.001$ ) (figure 2). This result did not change when additional potential covariates were adjusted for (table 2).

The age/sex-adjusted analysis of the percentage time spent within certain PA intensities showed that patients with HF spent a larger proportion of time sedentary and less time in light, moderate and vigorous PAs (figure $3 b$ ). The analysis of hourly mean acceleration values showed that patients with HF were significantly less active throughout the entire day (figure 3c). Between the hours of 06:00 and 19:00, participants without HF achieved 
Table 1 Baseline characteristics of UK Biobank participants

\begin{tabular}{|c|c|c|c|c|c|c|c|c|c|}
\hline & \multicolumn{3}{|c|}{ Self-reported PA } & \multicolumn{3}{|c|}{ Accelerometer-derived PA } & \multicolumn{3}{|c|}{ Overlapping PA } \\
\hline & No HF & HF & $P$ value & No HF & HF & $P$ value & No HF & HF & $\mathbf{P}$ value \\
\hline $\mathrm{n}$ & 387580 & 1600 & & 96105 & 596 & & 80121 & 190 & $<0.001$ \\
\hline Age (years) & $57(50-63)$ & $63(59-67)$ & $<0.001$ & $63(56-69)$ & $66(65-72)$ & $<0.001$ & $57(50-62)$ & $62(58-65)$ & $<0.001$ \\
\hline Female & $202169(52 \%)$ & $324(20 \%)$ & $<0.001$ & $54293(57 \%)$ & $153(26 \%)$ & & $43727(55 \%)$ & $28(15 \%)$ & \\
\hline Male & $185411(48 \%)$ & $1276(80 \%)$ & & $41812(44 \%)$ & $443(74 \%)$ & & $36394(46 \%)$ & $162(85 \%)$ & \\
\hline Ethnic background & & & 0.75 & & & 0.74 & & & 0.40 \\
\hline White & $367547(95 \%)$ & $1526(95 \%)$ & & $92803(97 \%)$ & $579(98 \%)$ & & $77490(97 \%)$ & $186(98 \%)$ & \\
\hline Mixed or non-white & $18915(5 \%)$ & $67(4 \%)$ & & $2968(3 \%)$ & $15(2 \%)$ & & $2413(3 \%)$ & $3(2 \%)$ & \\
\hline Missing & $1118(<1 \%)$ & $7(<1 \%)$ & & $334(<1 \%)$ & $2(<1 \%)$ & & $218(<1 \%)$ & $1(<1 \%)$ & \\
\hline BMI $\left(\mathrm{kg} / \mathrm{m}^{2}\right)$ & & & $<0.001$ & & & $<0.001$ & & & $<0.001$ \\
\hline$<24.9$ & $127352(33 \%)$ & $259(16 \%)$ & & $36815(38 \%)$ & $120(20 \%)$ & & $31033(39 \%)$ & $43(23 \%)$ & \\
\hline$\geq 25$ and $<30$ & 165702 (43\%) & $617(39 \%)$ & & $39488(41 \%)$ & $247(42 \%)$ & & $33073(41 \%)$ & 74 (39\%) & \\
\hline$\geq 30$ and $<35$ & $64782(17 \%)$ & $466(29 \%)$ & & $13736(14 \%)$ & $144(25 \%)$ & & $11209(14 \%)$ & $50(26 \%)$ & \\
\hline$\geq 35$ & $24159(6 \%)$ & $226(14 \%)$ & & $4810(5 \%)$ & 77 (13\%) & & $3783(5 \%)$ & $19(10 \%)$ & \\
\hline Missing & $5585(2 \%)$ & $32(2 \%)$ & & $1256(1 \%)$ & $8(1 \%)$ & & $1023(1 \%)$ & $4(2 \%)$ & \\
\hline Alcohol status & & & $<0.001$ & & & 0.003 & & & $<0.004$ \\
\hline Never & $14891(4 \%)$ & $94(6 \%)$ & & $2790(3 \%)$ & $27(5 \%)$ & & $2161(3 \%)$ & $6(3 \%)$ & \\
\hline Previous & $12987(3 \%)$ & $156(10 \%)$ & & $2620(3 \%)$ & $48(8 \%)$ & & $2118(3 \%)$ & $15(8 \%)$ & \\
\hline Current & 359513 (93\%) & $1349(84 \%)$ & & 90614 (94\%) & $521(87 \%)$ & & $75825(95 \%)$ & $169(89 \%)$ & \\
\hline Missing & $189(<1 \%)$ & $1(<1 \%)$ & & $81(<1 \%)$ & 0 & & $17(<1 \%)$ & 0 & \\
\hline Smoking status & & & $<0.001$ & & & 0.002 & & & $<0.002$ \\
\hline Never & 212488 (55\%) & $565(35 \%)$ & & $54839(57 \%)$ & $237(40 \%)$ & & $45656(57 \%)$ & 77 (41\%) & \\
\hline Previous & 134923 (35\%) & $842(53 \%)$ & & $34477(36 \%)$ & 307 (52\%) & & $28815(36 \%)$ & 101 (53\%) & \\
\hline Current & $39197(10 \%)$ & $189(12 \%)$ & & $6633(7 \%)$ & $50(8 \%)$ & & $5526(7 \%)$ & $12(6 \%)$ & \\
\hline Missing & $972(<1 \%)$ & 0 & & $156(<1 \%)$ & $2(<1 \%)$ & & $124(<1 \%)$ & 0 & \\
\hline Socioeconomic status & & & 0.06 & & & 0.87 & & & 0.13 \\
\hline Q1 & $77446(20 \%)$ & $213(13 \%)$ & & $21154(22 \%)$ & $110(18 \%)$ & & $17765(22 \%)$ & $27(14 \%)$ & \\
\hline Q2 & $77444(20 \%)$ & $272(17 \%)$ & & $20338(21 \%)$ & $130(22 \%)$ & & $16987(21 \%)$ & $51(27 \%)$ & \\
\hline Q3 & $77451(20 \%)$ & $285(18 \%)$ & & $19666(21 \%)$ & $121(20 \%)$ & & $16380(20 \%)$ & $33(17 \%)$ & \\
\hline Q4 & $77404(20 \%)$ & $330(21 \%)$ & & $18969(20 \%)$ & $117(20 \%)$ & & $15841(20 \%)$ & $50(26 \%)$ & \\
\hline Q5 & $77250(20 \%)$ & $497(31 \%)$ & & $15868(17 \%)$ & $118(20 \%)$ & & $13047(16 \%)$ & $29(15 \%)$ & \\
\hline Missing & $585(<1 \%)$ & 0 & & $110(<1 \%)$ & 0 & & $101(<1 \%)$ & 0 & \\
\hline
\end{tabular}

Quantile borders for the Townsend score were $-6.26,-3.97,-2.84,-1.44,1.11,11.00$ based on the participants with self-reported PA data.

Participants with poor wear quality and missing calibration coefficients are not included in the table. P values were calculated using the KolmogorovSmirnov test for continuous variables and the $\chi^{2}$ test for categorical variables. The overlapping group refers to individuals who were included in the self-reported analysis and also had sufficient-quality accelerometer recordings.

BMI, Body Mass Index; HF, heart failure; PA, physical activity.

approximately $20 \%$ higher mean acceleration per hour than HF participants when adjusting for age and sex.

Median times since first HF-related hospitalisation were 3.6 (95\% CI 1.6 to 6.1$)$ years for the self-reported analysis and 4.2 (95\% CI 2.0 to 8.2 ) years for the accelerometer analysis. Median times since last hospitalisation were 2.9 (95\% CI 1.2 to 5.5 ) years and 3.3 (95\% CI 1.3 to 6.9 ) years, respectively. The time since first report of $\mathrm{HF}$ or since last HF-related hospitalisation did not have a significant impact on the mean acceleration values measured (table 3). The results of the sensitivity analysis, restricting the comparisons to the overlapping group of participants who had HF at baseline and adjusting for age and sex, were broadly consistent with our main results (online supplementary figure $\mathrm{S} 1$ ).

\section{DISCUSSION}

Our analysis of PA levels in the UK Biobank population showed that both perceived and objectively measured PA levels of patients with HF were significantly reduced in comparison with people without HF.

The accelerometer-derived PA comparison showed that individuals with HF were on average $16 \%$ less physically active than individuals without a prior diagnosis of HF. This is in agreement with, although slightly lower 


\section{Baseline Analysis}

Overlapping Analysis

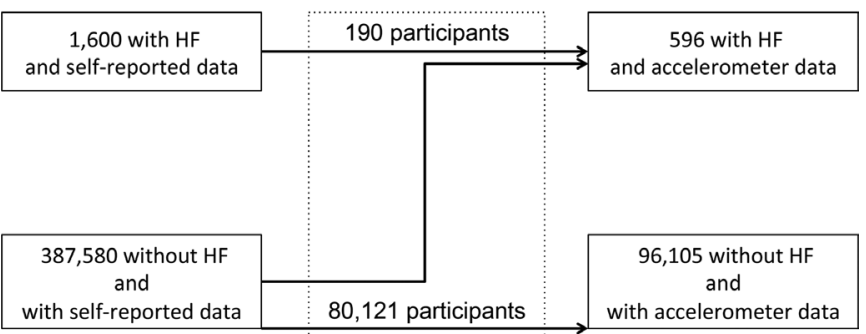

Figure 2 Flowchart of the participants included in the baseline and accelerometer analyses. Some participants included in the accelerometer analysis did not provide selfreported activity data at baseline; others developed HF in the period between baseline assessment and accelerometer recording. Participants falling into both the baseline and accelerometer analysis groups (overlapping analysis) were included in a separate sensitivity analysis. HF, heart failure.

than, previous findings from small-scale studies, which suggested that patients with $\mathrm{HF}$ are $44 \%-65 \%$ less physically active (based on step counts/movement-related activity) than individuals without HF. ${ }^{9} 132324$ Moreover, our study extends previous studies and shows that the average lower-level of PA was not due to a reduction in more intense types of activities but due to a generally less active lifestyle throughout the day, with peak differences occurring between 06:00 and 20:00. To our knowledge, this is the first study giving an insight into HF PA levels throughout the day.

Past studies have shown that self-reported PA levels are low to moderately correlated with objectively recorded PA levels ${ }^{25}$ and may be affected by socioeconomic
Table 3 Secondary analysis: adjusting for time since first and last hospitalisation

\begin{tabular}{|ll|}
\hline Model & $\begin{array}{l}\text { P value of 'time } \\
\text { since' variable }\end{array}$ \\
\hline $\begin{array}{l}\text { Self-reported } \\
\quad \text { Baseline+time since first diagnosis }\end{array}$ & 0.29 \\
\hline $\begin{array}{l}\text { My Baseline+time since last hospitalisation } \\
\quad \text { Baseline+time since last primary hospitalisation }\end{array}$ & 0.65 \\
\hline Accelerometer-derived & 0.91 \\
\hline $\begin{array}{l}\text { Within HF } \\
\text { Baseline+time since first diagnosis }\end{array}$ \\
\hline Baseline+time since last hospitalisation & 0.69 \\
\hline Baseline+time since last primary hospitalisation & 0.10 \\
\hline
\end{tabular}

The baseline model includes age, sex and HF status as input variables.

$\mathrm{HF}$, heart failure.

factors. ${ }^{27}$ Despite this, the general message of both the selfreported (perceived) and accelerometer-derived (objective) measurements investigated as part of this analysis was consistent. However, accelerometer recordings can provide a more detailed and precise assessment of activity levels of patients with HF throughout the day and over time.

PA levels of patients with $\mathrm{HF}$ within this cohort were not significantly affected by time after diagnosis of HF. These results contrast with findings by Miura $e t a l^{2}$ who studied self-reported PA levels in 4500 Japanese patients with HF and found that less than $30 \%$ of patients with HF managed to maintain their self-reported PA levels over a 1.4year time span. Both selection and survival bias intrinsic to

Table 2 Comparison of mean daily PA in patients with versus without HF with cumulative adjustments for covariates

\begin{tabular}{llll}
\hline Model & HF mean PA (CI) & No HF mean PA (Cl) & P value of HF status \\
\hline $\begin{array}{l}\text { Self-reported (in METs) } \\
\text { Baseline (age and sex, HF) }\end{array}$ & $26.5(24.7$ to 28.4$)$ & $34.7(34.5$ to 34.9$)$ & $<0.001$ \\
\hline Baseline+ethnicity & $26.6(24.7$ to 28.4$)$ & $34.7(34.5$ to 34.9$)$ & $<0.001$ \\
\hline Baseline+BMI & $28.2(26.4$ to 30.1$)$ & $34.7(34.5$ to 34.9$)$ & $<0.001$ \\
\hline Baseline+alcohol status & $26.5(24.6$ to 28.3$)$ & $34.7(34.5$ to 34.9$)$ & $<0.001$ \\
\hline Baseline+smoking status & $26.4(24.5$ to 28.2$)$ & $34.7(34.5$ to 34.9$)$ & $<0.001$ \\
\hline Baseline+Townsend & $26.1(24.3$ to 28.0$)$ & $34.7(34.5$ to 34.9$)$ & $<0.001$ \\
\hline Baseline+all of the above & $27.7(25.8$ to 30.0$)$ & $34.7(34.4$ to 35.0$)$ & $<0.001$ \\
\hline Accelerometer-derived (in milligravity) & & $28.1(28.0$ to 28.1$)$ & $<0.001$ \\
\hline Baseline (age and sex, HF) & $23.7(23.1$ to 24.4$)$ & $28.1(28.0$ to 28.2$)$ & $<0.001$ \\
\hline Baseline+ethnicity & $23.7(23.1$ to 24.4$)$ & $28.1(28.0$ to 28.2$)$ & $<0.001$ \\
\hline Baseline+BMl & $24.7(24.0$ to 25.3$)$ & $28.1(28.0$ to 28.1$)$ & $<0.001$ \\
\hline Baseline+alcohol status & $23.7(23.1$ to 24.4$)$ & $28.1(28.0$ to 28.2$)$ & $<0.001$ \\
\hline Baseline +smoking status & $23.7(23.1$ to 24.4$)$ & $28.1(28.0$ to 28.2$)$ & $<0.001$ \\
\hline Baseline+Townsend & $23.8(23.1$ to 24.4$)$ & $28.1(27.9$ to 28.2$)$ & $<0.001$ \\
\hline Baseline+all of the above & $24.7(24.1$ to 25.4$)$ & & \\
\hline
\end{tabular}

$P$ values are reported for $\mathrm{HF}$ status adjusted for the listed covariates.

BMI, Body Mass Index; HF, heart failure; MET, metabolic equivalent of task; PA, physical activity. 


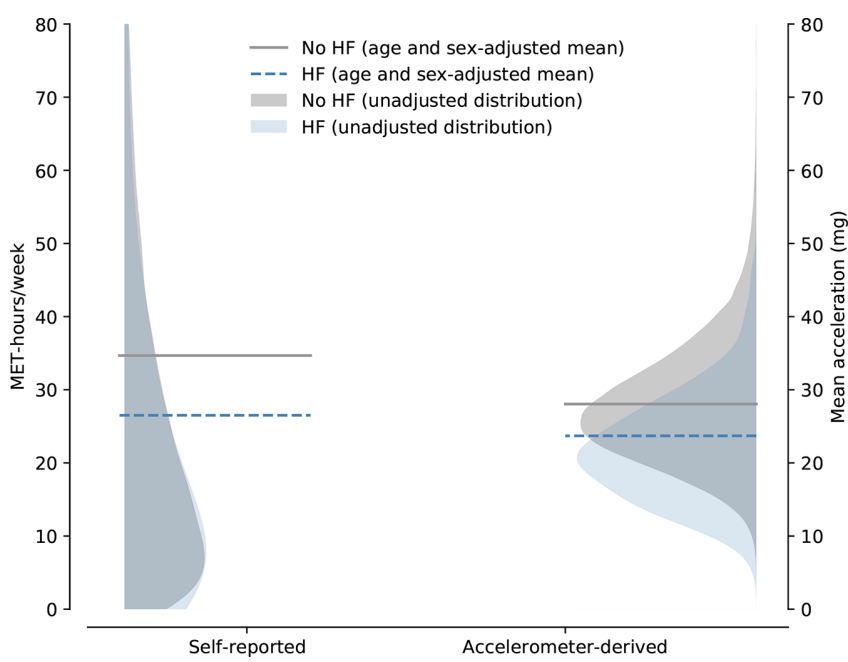

Figure 3 Comparison of self-reported $(p<0.001)$ and accelerometer-derived $(p<0.001)$ physical activityPA in participants with or without heart failureHF. Shaded areas depict unadjusted physical activityPA distributions and dashed lines show age-adjusted/sex-adjusted mean physical activityPA. HF, heart failure; MET, metabolic equivalent of task; PA, physical activity.

the UK Biobank accelerometer data might have distorted the measured effect of time since first and last HF-related hospitalisations on PA levels. Further studies will therefore be needed to quantify the degree and determinants of change in PA over time.

Limitations of this study include its cross-sectional nature and the fact that both the self-reported and accelerometer data provided by the UK Biobank might be affected by selection bias, resulting in a healthier than normal study population represented in this analysis. ${ }^{28}$ This would lead to a sample not representative of the HF population at large. Thus, our findings may be more applicable to patients with less severe chronic HF.

In summary, our results show that perceived and objectively recorded PA levels of patients with chronic HF are significantly lower than those of individuals without HF. This difference is continuous throughout the different hours of the day and suggests that increases in everyday activity may be a potential alternative to structured exercise programmes.

Acknowledgements We thank all UK Biobank participants for agreeing to volunteer in this research.

Contributors JOD, KR and LT designed the project. JOD and KS-B performed the analysis of the data. CV, NC, GS-K and AD provided support on the analysis of the data. KR and TD provided support on the interpretation of the data. JOD wrote the paper with input from all authors.

Funding This work was supported by the National Institute of Health Research, Oxford Biomedical Research Centre (AD and KR) and the Oxford Martin School (KR and GS-K). JOD acknowledges the support of the RCUK Digital Economy Programme (grant number EP/G036861) (Oxford Center for Doctoral Training in Healthcare Innovation, UK). AD is also supported by the British Heart Foundation Centre of Research Excellence at Oxford, UK (grant number RE/13/1/30181).

Disclaimer No funding bodies had any role in the analysis, decision to publish, or preparation of the manuscript.

Competing interests None declared.
Patient consent for publication Not required.

Ethics approval The study was covered by the general ethics approval for UK Biobank studies from the NHS National Research Ethics Service (ref 11/NW/0382).

Provenance and peer review Not commissioned; internally peer reviewed.

Data availability statement Data may be obtained from a third party and are not publicly available. Data cannot be shared publicly and is subject to a license agreement. Researchers who wish to obtain access to data should contact the UK Biobank Resource (access@ukbiobank.ac.uk).

Open access This is an open access article distributed in accordance with the Creative Commons Attribution 4.0 Unported (CC BY 4.0) license, which permits others to copy, redistribute, remix, transform and build upon this work for any purpose, provided the original work is properly cited, a link to the licence is given, and indication of whether changes were made. See: https://creativecommons.org/ licenses/by/4.0/.

\section{ORCID iDs}

Johanna O'Donnell http://orcid.org/0000-0001-8028-8224

Nathalie Conrad http://orcid.org/0000-0001-5027-5481

Kazem Rahimi http://orcid.org/0000-0001-9895-1493

\section{REFERENCES}

1 Lee H, Boo S, Yu J, et al. Physical functioning, physical activity, exercise self-efficacy, and quality of life among individuals with chronic heart failure in Korea: a cross-sectional descriptive study. $J$ Nurs Res 2017;25:131-9.

2 Miura Y, Fukumoto Y, Miura T, et al. Impact of physical activity on cardiovascular events in patients with chronic heart failure. A multicenter prospective cohort study. Circ J 2013;77:2963-72.

3 Kondamudi N, Haykowsky M, Forman DE, et al. Exercise training for prevention and treatment of heart failure. Prog Cardiovasc Dis 2017;60:115-20.

4 Piepoli MF, Conraads V, Corrà U, et al. Exercise training in heart failure: from theory to practice. A consensus document of the heart failure association and the European association for cardiovascular prevention and rehabilitation. Eur J Heart Fail 2011;13:347-57.

5 National Institute for Health and Care Excellence. Chronic heart failure in adults: management | Guidance and guidelines. [Internet]. Available: https://www.nice.org.uk/guidance/cg108/chapter/1guidance [Accessed 26 Jun 2017].

6 Yancy CW, Jessup M, Bozkurt B, et al. 2013 ACCF/AHA guideline for the management of heart failure: a report of the American College of cardiology Foundation/American heart association Task force on practice guidelines. Circulation 2013;128:e240-327.

7 Conraads VM, Deaton C, Piotrowicz E, et al. Adherence of heart failure patients to exercise: barriers and possible solutions. Eur J Heart Fail 2012;14:451-8.

8 Tierney S, Elwers H, Sange C, et al. What influences physical activity in people with heart failure?: a qualitative study. Int J Nurs Stud 2011;48:1234-43.

9 van den Berg-Emons HR, Bussmann JH, Balk A, et al. Level of activities associated with mobility during everyday life in patients with chronic congestive heart failure as measured with an "activity monitor". Phys Ther 2001;81:1502-11.

10 Jehn M, Schmidt-Trucksäss A, Schuster T, et al. Daily walking performance as an independent predictor of advanced heart failure: prediction of exercise capacity in chronic heart failure. Am Heart $J$ 2009;157:292-8.

11 Izawa KP, Watanabe S, Oka K, et al. Physical activity in relation to exercise capacity in chronic heart failure patients. Int $J$ Cardiol 2011;152:152-3.

12 Izawa KP, Watanabe S, Oka K, et al. Usefulness of step counts to predict mortality in Japanese patients with heart failure. Am J Cardiol 2013;111:1767-71.

13 Walsh JT, Charlesworth A, Andrews R, et al. Relation of daily activity levels in patients with chronic heart failure to long-term prognosis. Am J Cardiol 1997;79:1364-9.

14 Sudlow C, Gallacher J, Allen N, et al. UK Biobank: an open access resource for identifying the causes of a wide range of complex diseases of middle and old age. PLoS Med 2015;12:e1001779.

15 Craig CL, Marshall AL, Sjöström M, et al. International physical activity questionnaire: 12 -country reliability and validity. Med Sci Sports Exerc 2003;35:1381-95.

16 Bradbury KE, Guo W, Cairns BJ, et al. Association between physical activity and body fat percentage, with adjustment for BMI: a large cross-sectional analysis of UK Biobank. BMJ Open 2017;7:e011843. 
17 Sjostrom M, Ainsworth B, Bauman A. Guidelines for data processing analysis of the International Physical Activity Questionnaire (IPAQ) Short and long forms. IPAQ, 2005.

18 Doherty A, Jackson D, Hammerla N, et al. Large scale population assessment of physical activity using wrist worn Accelerometers: the UK Biobank study. PLoS One 2017;12:e0169649.

19 van Hees VT, Fang Z, Langford J, et al. Autocalibration of accelerometer data for free-living physical activity assessment using local gravity and temperature: an evaluation on four continents. $J$ Appl Physiol 2014;117:738-44.

20 Hildebrand M, Van Hees VT, Hansen BH, et al. Age group comparability of raw accelerometer output from wrist- and hip-worn monitors. Med Sci Sports Exerc 2014;46:1816-24.

21 Townsend P, Phillimore P, Beattie A. Health and deprivation: inequality and the North. Croom Helm, 1988: 250.

22 R Development Core Team. R: A language and environment for statistical computing. [Internet]. Vienna, Austria: R Foundation for Statistical Computing, 2008. http://www.R-project.org
23 Hoodless DJ, Stainer K, Savic N, et al. Reduced customary activity in chronic heart failure: assessment with a new shoe-mounted pedometer. Int J Cardiol 1994;43:39-42.

24 Barker J, Smith Byrne K, Doherty A, et al. Physical activity of UK adults with chronic disease: cross-sectional analysis of accelerometer-measured physical activity in 96706 UK Biobank participants. Int J Epidemiol 2019;48:1167-74.

25 Prince SA, Adamo KB, Hamel ME, et al. A comparison of direct versus self-report measures for assessing physical activity in adults: a systematic review. Int J Behav Nutr Phys Act 2008;5.

26 Pozehl B, Duncan K, Norman J, et al. Daily physical activity levels as measured by self-report and Accelerometry in patients with heart failure. J Card Fail 2009;15:S99.

27 Sabia S, van Hees VT, Shipley MJ, et al. Association between questionnaire- and accelerometer-assessed physical activity: the role of sociodemographic factors. Am J Epidemiol 2014;179:781-90.

28 Littlejohns TJ, Sudlow C, Allen NE, et al. UK Biobank: opportunities for cardiovascular research. Eur Heart J 2019;40:1158-66. 